\title{
Growth estimates for generalized factors of $H^{p}$ spaces
}

\author{
by \\ Joseph A. Cima (Chapel Hill, NC), Angeliki Kazas (Oneonta, NY) \\ and Michael I. Stessin (Albany, NY)
}

\begin{abstract}
With $\varphi$ an inner function and $M_{\varphi}$ the multiplication operator on a given Hardy space it is known that for any given function $f$ in the Hardy space we may use the Wold decomposition to obtain a factorization of the given $f$ (not the Riesz factorization). This new factorization has been shown to be useful in the study of commutants of Toeplitz operators.

We study the smoothness of each factor of this factorization. We show in some cases that the factors lie in the same Hardy space (or smoothness class) as the given function $f$. We also construct an example to show that there are bounded, holomorphic functions which have factors that are not in a given Hardy $p$-space. Many of our results are produced by studying a natural class of positive measures associated to the given inner function.
\end{abstract}

1. Introduction. In the study of the classical theory of Hardy spaces $\left(H^{p}(\mathbb{D})\right)$ on the unit disk it has been shown that each function $f$ in such a space has a unique (up to a multiplicative unimodular constant) factorization. The factorization is given as a product of two holomorphic functions. The inner part of the factorization is a holomorphic function in the unit sphere of $H^{\infty}$ which incorporates the zeros of $f$ and its "singular" boundary behavior, whereas the outer part is nonzero and in the same $H^{p}$ space as the original $f$ (see [4]). Both parts of this type of factorization are important as function classes in their own right. For example the zeros of the function contained in the inner part must satisfy a geometric growth condition (the Blaschke condition) and the nonzero outer parts have the property that they are cyclic for the shift operator.

Recently, new research has been initiated in the study of the Wold decomposition for an analytic Toeplitz operator $T_{\varphi}$ on $H^{p}(\mathbb{D})$ spaces (see [6], [10]). For our interests the symbol $\varphi$ in the expression for the Toeplitz operator is a classical inner function. From this work it is possible to define another factorization of $H^{p}(\mathbb{D})$ functions that is more intrinsic to the Wold decomposition. In this factorization the terms depend on the inner func-

2000 Mathematics Subject Classification: 30D55, 47B35. 
tion $\varphi$ as follows. A function $h_{p}$ is said to be $\varphi-p$ inner if it satisfies:

(i) $\int_{-\pi}^{\pi}\left|h_{p}\left(e^{i t}\right)\right|^{p} d t=1$,

(ii) $\int_{0}^{2 \pi}\left|h_{p}\left(e^{i t}\right)\right|^{p} \varphi\left(e^{i t}\right)^{n} d t=0, n>0$.

Then for each $f \in H^{p}(\mathbb{D})$, there exists a factorization

$$
f=h_{p} F_{p} \circ \varphi
$$

where $F_{p}$ is an outer function in $H^{p}$, and $h_{p}$ is $\varphi$-p inner. We call this the $\varphi-p$ factorization of $f$ (see [6], and Section 2.1 below). One can see the efficacy of this factorization in various aspects of function theory and its relationship to the natural operators acting on these spaces. For example, the description of the commutant of the operator of multiplication by an inner function $\varphi$ can be given in terms of this factorization (see [10]).

In addition to the Wold decomposition, another tool is very useful in studying properties of such $\varphi-p$ inner functions. By the Herglotz theorem it is known that for a complex number $w$ of modulus one there is a nonnegative singular measure $\sigma_{w}$ on the unit circle $\mathbb{T}$ such that

$$
\operatorname{Re} \frac{w+\varphi(z)}{w-\varphi(z)}=\int_{\mathbb{T}} \operatorname{Re} \frac{\zeta+z}{\zeta-z} d \sigma_{w}(\zeta), \quad z \in \mathbb{D} .
$$

These measures were introduced by Clark in 1972, in his study of onedimensional perturbations of the shift operators (see [3]). Further properties of these measures were investigated by Aleksandrov and Poltoratskiü [1], [8]).

The study of the properties of these measures leads to a more complete understanding of the properties of $\varphi-p$ inner and outer functions. In particular using a result of A. B. Aleksandrov an explicit integral formula has been given for the $\varphi-p$ outer part of the factorization (see [10], and Section 2 below).

It was proven in [6] that if $\varphi$ is a finite Blaschke product then the $\varphi-p$ inner part of any function is bounded. In general, this result does not hold (see [6], [10], and Section 5 below). However, for certain $f \in H^{p}$ we can say the $\varphi-p$ inner part is bounded for all $\varphi$. For example, assume $\varphi$ is not a finite Blaschke product and that $f$ is analytic in the closed unit disk. Then for every inner function $\varphi$, the $\varphi$ - $p$ inner part $h_{p}$ of $f$ is bounded. Indeed, assume that $h_{p}$ is unbounded. Then there exists a sequence $\left\{w_{k}\right\} \subset \mathbb{T}$ such that $\varphi$ has nontangential limit of modulus 1 for all $w_{k}$, and $\lim _{k \rightarrow \infty}\left|h_{p}\left(w_{k}\right)\right|=\infty$. Let $\lim _{k \rightarrow \infty} \varphi\left(w_{k}\right)=\tau$. Then

$$
\int_{\mathbb{T}}|f(\zeta)|^{p} d \sigma_{\tau}(\zeta)=0
$$

(The proof of this statement is similar to the one of Lemma 2 in Section 3.) Since $\sigma_{\tau}$ is a nonnegative measure, this implies that $f$ vanishes on the carrier of $\sigma_{\tau}$. Being analytic in the closed unit disk, $f$ has only a finite number of 
zeros on the unit circle. Thus the carrier of $\sigma_{\tau}$ is finite. By Aleksandrov's theorem (Theorem D, Section 2.2), $\varphi$ has to be a finite Blaschke product, a contradiction. Theorem 1 below gives a more general result.

If $\varphi$ is inner, then $\varphi(z)=B(z) S(z)$ where $B$ is a Blaschke product with zeros $\left\{a_{n}\right\}$ and $S$ is a singular inner function generated by the singular measure $\mu$. Following the notation of Nikol'skiı (see [7, p. 62]), we let

$$
\Sigma_{\varphi}=\operatorname{supp}(\mu) \cup \overline{\left\{a_{1}, \ldots, a_{n}, \ldots\right\}}
$$

denote the spectrum of $\varphi$.

THEOREM 1. Let $\varphi$ be an inner function. If $f \in H^{p}$, and $f$ is analytically extendable through a neighborhood of $\Sigma_{\varphi} \cap \mathbb{T}$, then the $\varphi$-p inner part of $f$ is bounded.

To set the strong assumption that $f$ is analytically extendable through the spectrum in $\mathbb{T}$ of $\varphi$ in perspective we note that there are examples of $f$ in $H^{\infty}$ whose $\varphi$-p inner part is not in $H^{\infty}$. An example of such behavior is given in [10]. In that particular example, the $\varphi$-p inner part has logarithmic growth. In Section 5 below, we further investigate this phenomenon and strengthen the result in [10]. The general idea of the proof is analogous to [10] but we modify the construction to obtain sharper estimates. We prove the following theorem.

THEOREM 2. Let $\varphi$ be an inner function having the property that for all $w \in \mathbb{T}, \sigma_{w}$ is a continuous measure. There exists a function $f: \mathbb{D} \rightarrow \mathbb{D}$ in $H^{\infty}$ such that for any $p, 0<p \leq \infty$, the $\varphi$-p inner part of $f$ is not in $L^{q}$ for any $q>p$.

We can find functions $\varphi$ for which the associated measures $\sigma_{w}$ are continuous. Consider, for example, a sequence of contiguous arcs $I_{n}$ in $\mathbb{T}$ where $\left|I_{n}\right|=1 / n$. Let $\zeta_{n}$ be the midpoint of $I_{n}$ and define $a_{n}=\left(1-1 / n^{2}\right) \zeta_{n}$. If $B(z)$ is the infinite Blaschke product with zeros $a_{n}$, then $B$ is an inner function such that $\sigma_{w}$ is continuous for all $w \in \mathbb{T}$ (see [9, p. 185] for details).

The assumption of continuity of the $\sigma_{w}$ measures is not necessary. For instance, in Section 5, we give an example which shows that a result similar to Theorem 2 holds when $\varphi$ is a singular inner function determined by a point mass at 1 . In this case the measures are countable sums of point masses.

It is natural to ask whether some special properties of $\varphi$ would guarantee that the $\varphi-p$ inner part is bounded for some wide classes of functions. We prove this is the case for all disk algebra functions, provided the spectrum of $\varphi$ contains an arc of the unit circle. For $|w|=1$, define the set $E_{w}=\{\zeta \in \mathbb{T}$ : $\varphi(\zeta)=w\}$. Here $\varphi(\zeta)$ denotes the nontangential unimodular limit of $\varphi$ at $\zeta$ (which exists for almost all $\zeta \in \mathbb{T}$ ). The following theorem is proved in Section 7 . 
TheORem 3. Let $\varphi$ be an inner function. Assume that there exists an arc $I \subset \mathbb{T}$ and $a w \in \mathbb{T}$ such that $E_{w}$ is dense in $I$. Let $f \in \mathbf{A}(f \neq 0)$. If $f=h_{p} F_{p} \circ \varphi$ is the $\varphi$-p factorization of $f$, then $h_{p}$ is bounded.

The assumption that $E_{w}$ is dense in some arc of the unit circle is natural as Theorem 4 illustrates.

THEOREM 4. Let $\varphi$ be an inner function which is not a finite Blaschke product. Assume that for some $w_{0} \in \mathbb{T}, E_{w_{0}}$ is nowhere dense in $\mathbb{T}$. Then there exists a function $f \in \mathbf{A}$ whose $\varphi$-p inner part is unbounded.

The structure of the paper is as follows. In Section 2, we present all necessary background results. We give results on generalized factorization, and define and list properties of singular measures associated with an inner function. Here we state the relevant theorems of Aleksandrov and show the relation of $\varphi-p$ inner and outer functions to these singular measures. In Section 3, we present two auxiliary results which relate the singular measures associated with the composition of two inner functions. In Section 4, we prove some smoothness results about the behavior of the outer part of the $\varphi-p$ factorization. Here, the behavior of the $\varphi$ - $p$ outer functions is consistent with the behavior of outer functions in the classical factorization of $H^{p}$ functions. In Section 5, we show that a function $f \in H^{\infty}$ can have a $\varphi-p$ inner part which is unbounded, for any $\varphi$ with continuous $\sigma_{w}$ measures. This result is sharp in the sense that we can produce functions $f \in H^{\infty}$ whose $\varphi$-p inner part $(p<\infty)$ is not in $L^{q}$ for any $q>p$. In Section 6 , we prove Theorem 1 stated above. Theorems 3 and 4 are proved in Section 7.

Acknowledgements. The authors would like to thank the referee for his comments.

\section{Background results}

2.1. Generalized factorization. The definitions of inner and outer functions, Blaschke products, singular functions, and Hardy spaces can be found in standard texts (see for example [4]) and we refer the reader to these works.

If $f \in H^{p}$ and $\varphi$ is inner, the following theorem was proved in [6].

Theorem A. Let $p>0$. There exists a factorization

$$
f=h_{p} F_{p} \circ \varphi
$$

where $F_{p}$ is an outer function in $H^{p}$, and $h_{p}$ is $\varphi$-p inner. The $\varphi$-p factorization is unique up to a unimodular factor.

The factors $h_{p}$ and $F_{p}$ are called the $\varphi-p$ inner and $\varphi$ - $p$ outer parts of the factorization respectively. When $\varphi(z)=z$, the $\varphi$ - $p$ factorization above coincides with the classical factorization of $f$ into its inner and outer parts. 
For $f \in H^{p} \subset H^{p^{\prime}}$ (i.e. $p^{\prime}<p$ ) this factorization produces different factors in contrast to the classical case.

2.2. Singular measures associated with an inner function. Let $|w|=1$. Then

$$
\operatorname{Re} \frac{w+\varphi(z)}{w-\varphi(z)}>0
$$

for all $z$ in the unit disk. By Herglotz's theorem there is a nonnegative measure $\sigma_{w}^{\varphi}$ on the unit circle $\mathbb{T}$ such that

$$
\operatorname{Re} \frac{w+\varphi(z)}{w-\varphi(z)}=\int_{\mathbb{T}} \operatorname{Re} \frac{\zeta+z}{\zeta-z} d \sigma_{w}^{\varphi}(\zeta)
$$

for all $z \in \mathbb{D}$. Since $\varphi$ is inner, $\sigma_{w}^{\varphi}$ is singular with respect to the normalized Lebesgue measure $d m$ on $\mathbb{T}$. In this paper, if it is clear which function $\varphi$ the measure is associated with, we will omit the superscript $\varphi$. It was proven by Aleksandrov (see [1]) that if $E_{w}$ is the set of points in $\mathbb{T}$ where $\varphi$ has $w$ as a nontangential limit, then

$$
\sigma_{w}\left(\mathbb{T} \backslash E_{w}\right)=0 .
$$

If we set $z=0$ in (1), then we have

$$
\sigma_{w}(\mathbb{T})=\frac{1-|\varphi(0)|^{2}}{|1-\bar{w} \varphi(0)|^{2}},
$$

from which we can obtain

$$
\frac{1-|\varphi(0)|}{1+|\varphi(0)|} \leq \sigma_{w}(\mathbb{T}) \leq \frac{1+|\varphi(0)|}{1-|\varphi(0)|} .
$$

In particular, if $\varphi(0)=0$, then $\sigma_{w}$ is a probability measure for all $w \in \mathbb{T}$.

From (1) one can obtain the following formula for the Fourier coefficients of the $\sigma_{w}$ measures (see [1]):

$$
\int_{\mathbb{T}} \zeta^{-n} d \sigma_{w}(\zeta)= \begin{cases}\sum_{k>0} \overline{w^{k}} \int_{\mathbb{T}} \varphi(\zeta)^{k} \zeta^{-n} d m(\zeta), & n>0, \\ \frac{1-|\varphi(0)|^{2}}{|1-\bar{w} \varphi(0)|^{2}}, & n=0 .\end{cases}
$$

As a consequence of this equality, Aleksandrov obtained the following theorem for any Lebesgue integrable function defined on the unit circle (see [1]).

Theorem B. If $f: \mathbb{T} \rightarrow \mathbb{C}$ and $f \in L^{1}(\mathbb{T}, d m)$, then $f \in L^{1}\left(\mathbb{T}, d \sigma_{w}\right)$ for almost all $w \in \mathbb{T}$. Furthermore,

$$
\int_{\mathbb{T}} f(\zeta) d m(\zeta)=\iint_{\mathbb{T}} f(\zeta) d \sigma_{w}(\zeta) d m(w) .
$$

REMARK. As a corollary to Theorem B, we obtain the following well known result. If $\varphi$ is an inner function and $\varphi(0)=0$, then $\varphi$ is a measure 
preserving mapping of the circle into itself. Indeed, consider $V=\varphi^{-1}(I)=$ $\bigcup_{w \in I} \varphi^{-1}(\{w\})$ where $I$ is a Lebesgue measurable set. If $\varphi(0)=0$, then $\sigma_{w}$ is a probability measure for every $w \in \mathbb{T}$, and by Theorem $\mathrm{B}$,

$$
\begin{aligned}
|V| & =\int_{\mathbb{T}} \chi_{V}(\zeta) d m(\zeta)=\iint_{\mathbb{T}} \chi_{V}(\zeta) d \sigma_{w}(\zeta) d m(w) \\
& =\int_{\mathbb{T}} \chi_{I}(w) d m(w)=|I| .
\end{aligned}
$$

If $\varphi(0) \neq 0$, then $(3)$ and (5) imply that

$$
\frac{1-|\varphi(0)|}{1+|\varphi(0)|}|I| \leq|V| \leq \frac{1+|\varphi(0)|}{1-|\varphi(0)|}|I| .
$$

Recall that, for each self-mapping of the unit disk, $\varphi: \mathbb{D} \rightarrow \mathbb{D}$, the angular derivative $D_{\varphi}$ is defined as

$$
D_{\varphi}(\zeta)= \begin{cases}\lim _{r \rightarrow 1-} \frac{1-|\varphi(r \zeta)|^{2}}{1-r^{2}} & \text { if } \varphi(\zeta) \in \mathbb{T}, \\ \infty & \text { otherwise }\end{cases}
$$

(see [1]). If $\varphi$ is analytically extendable through some point $w \in \mathbb{T}$ then

$$
D_{\varphi}(\zeta)=\left|\varphi^{\prime}(\zeta)\right|
$$

Let

$$
E=\{w \in \mathbb{T}: \varphi(z) \text { has nontangential limit at } w \text { of modulus } 1\} .
$$

The following result is due to A. Aleksandrov (see [1]).

Theorem C. If $\zeta \in E$ and $w=\varphi(\zeta)$ then

$$
D_{\varphi}(\zeta)=\frac{1}{\sigma_{w}(\{\zeta\})} .
$$

In particular, $\sigma_{w}$ is continuous at $\zeta$ if and only if

$$
D_{\varphi}(\zeta)=\infty
$$

For $\varphi$ inner, and the set $E_{w}$ finite for some $w \in \mathbb{T}$, Aleksandrov proved the following (see [1]).

THEOREM D. If $\varphi$ is an inner function such that $E_{w}$ is finite for some $w \in \mathbb{T}$, then $\varphi$ is a finite Blaschke product.

The measures $\sigma_{w}$ are a convenient tool in dealing with the $\varphi-p$ factorization. The following theorems are proved in [10].

Theorem E. A function $h$ is $\varphi-p$ inner if and only if

$$
\int_{\mathbb{T}}|h(\zeta)|^{p} d \sigma_{w}(\zeta)=1
$$

for almost every $w \in \mathbb{T}$. 
Theorem F. Let $\varphi(z)=B(z) S(z)$, where $B$ is a Blaschke product and $S$ is a singular inner function generated by a singular measure $\mu$. If $w \notin$ $\Sigma_{\varphi} \cap \mathbb{T}$, then any $\varphi$-p inner function is bounded near $w$.

Theorem G. If $0<p<\infty, f \in H^{p}$, and $f=h_{p} F_{p} \circ \varphi$ is the $\varphi$ - $p$ factorization of $f$, then for $z \in \mathbb{D}$, the outer part $F_{p}$ is given by

$$
F_{p}(z)=\exp \left\{\frac{1}{p} \int_{\mathbb{T}} \frac{\zeta+z}{\zeta-z} \log \left(\int_{\mathbb{T}}|f(\tau)|^{p} d \sigma_{\zeta}(\tau)\right) d m(\zeta)\right\} .
$$

EXAMPLE. Let $f=z+z^{2}$, and let $\varphi$ be an inner function with $\varphi(0)=0$. If $f=h_{2} F_{2} \circ \varphi$, then we want to find the factors $F_{2}$ and $h_{2}$. From Theorem G, we have

$$
\begin{aligned}
F_{2}(z) & =\exp \left\{\int_{\mathbb{T}} \frac{\zeta+z}{\zeta-z} \log \left(\int_{\mathbb{T}}\left|\tau+\tau^{2}\right|^{2} d \sigma_{\zeta}(\tau)\right)^{1 / 2} d m(\zeta)\right\} \\
& =\exp \left\{\int_{\mathbb{T}} \frac{\zeta+z}{\zeta-z} \log \left(\int_{\mathbb{T}}(2+\tau+\bar{\tau}) d \sigma_{\zeta}(\tau)\right)^{1 / 2} d m(\zeta)\right\} .
\end{aligned}
$$

By (4) we obtain

$$
\left|F_{2}(\zeta)\right|^{2}=\int_{\mathbb{T}}(2+\tau+\bar{\tau}) d \sigma_{\zeta}(\tau)=2+\zeta \overline{\varphi^{\prime}(0)}+\bar{\zeta} \varphi^{\prime}(0) .
$$

It is easy to see that

$$
\left|F_{2}(\zeta)\right|^{2}=|a+b \zeta|^{2}
$$

where if $F_{2}$ is outer,

$$
a=\left(1+\left(1-\left|\varphi^{\prime}(0)\right|^{2}\right)^{1 / 2}\right)^{1 / 2}, \quad b=\frac{\overline{\varphi^{\prime}(0)}}{\left(1+\left(1-\left|\varphi^{\prime}(0)\right|^{2}\right)^{1 / 2}\right)^{1 / 2}} .
$$

Thus,

$$
\begin{aligned}
& F_{2}(\zeta)=\left(1+\left(1-\left|\varphi^{\prime}(0)\right|^{2}\right)^{1 / 2}\right)^{1 / 2}+\frac{\overline{\varphi^{\prime}(0)} \zeta}{\left(1+\left(1-\left|\varphi^{\prime}(0)\right|^{2}\right)^{1 / 2}\right)^{1 / 2}} \\
& h_{2}(\zeta)=\left(1+\left(1-\left|\varphi^{\prime}(0)\right|^{2}\right)^{1 / 2}\right)^{1 / 2} \frac{\zeta+\zeta^{2}}{\left.1+\left(1-\left|\varphi^{\prime}(0)\right|\right)^{2}\right)^{1 / 2}+\overline{\varphi^{\prime}(0)} \varphi(\zeta)} .
\end{aligned}
$$

3. Two auxiliary lemmas. The following lemma expresses singular measures associated with the composition of two inner functions.

Lemma 1. Let $\psi$ and $\chi$ be inner functions and let $\varphi=\psi \circ \chi$. Then

$$
\sigma_{w}^{\varphi}=\int_{\mathbb{T}} \sigma_{\tau}^{\chi} d \sigma_{w}^{\psi}(\zeta)
$$

That means that for every continuous function $f$ on $\mathbb{T}$,

$$
\int_{\mathbb{T}} f(\zeta) d \sigma_{w}^{\varphi}(\zeta)=\int_{\mathbb{T}} \int_{\mathbb{T}} f(\zeta) d \sigma_{\tau}^{\chi}(\zeta) d \sigma_{w}^{\psi}(\tau)
$$


Proof. Let $\zeta \in \mathbb{T}$. Consider the bounded linear functional

$$
L(f)=\iint_{\mathbb{T}} f(\zeta) d \sigma_{\tau}^{\chi}(\zeta) d \sigma_{w}^{\psi}(\tau)
$$

on the space of continuous functions on $\mathbb{T}$. By the Riesz theorem, there exists a measure $\mu$ such that

$$
L(f)=\int_{\mathbb{T}} f(\zeta) d \mu(\zeta)
$$

Fix $z \in \mathbb{D}$. Since

$$
f(\tau)=\operatorname{Re} \frac{\tau+z}{\tau-z}
$$

is continuous we have

$$
\begin{aligned}
\int_{\mathbb{T}} \operatorname{Re} \frac{\zeta+z}{\zeta-z} d \mu(\zeta) & =\iint_{\mathbb{T}} \operatorname{Re} \frac{\zeta+z}{\zeta-z} d \sigma_{\tau}^{\chi}(\zeta) d \sigma_{w}^{\psi}(\tau)=\int_{\mathbb{T}} \operatorname{Re} \frac{\tau+\chi(z)}{\tau-\chi(z)} d \sigma_{w}^{\psi}(\tau) \\
& =\operatorname{Re} \frac{w+\varphi(z)}{w-\varphi(z)}=\int_{\mathbb{T}} \operatorname{Re} \frac{\zeta+z}{\zeta-z} d \sigma_{w}^{\varphi}(\zeta) .
\end{aligned}
$$

By the Uniqueness Theorem $\mu=\sigma_{w}^{\varphi}$.

Corollary 1. Let $\varphi$ be an inner function and $a \in \mathbb{T}$. If

$$
\varphi_{1}(z)=\frac{\varphi(z)-a}{1-\bar{a} \varphi(z)}
$$

then

$$
\sigma_{w}^{\varphi_{1}}=\frac{1-|a|^{2}}{|1+\bar{a} w|^{2}} \sigma_{w_{1}}^{\varphi}
$$

where

$$
w_{1}=\frac{w+a}{1+\bar{a} w} .
$$

Proof. Let

$$
\psi(z)=\frac{z-a}{1-\bar{a} z} .
$$

Then $\varphi_{1}=\psi \circ \varphi$. Now the result follows since

$$
\sigma_{w}^{\psi}=\frac{\left|1-\bar{a} w_{1}\right|^{2}}{1-|a|^{2}} \delta_{w_{1}}=\frac{1-|a|^{2}}{|1+\bar{a} w|^{2}} \delta_{w_{1}} .
$$

The next result shows that the family of $\sigma_{w}$ measures is weak-* continuous.

Lemma 2. Let $\varphi$ be an inner function. If $\left\{w_{n}\right\}$ is a sequence in $\mathbb{T}$ with $\lim _{n \rightarrow \infty} w_{n}=w$ then for any continuous function $f$ on $\mathbb{T}$,

$$
\lim _{n \rightarrow \infty}\left\{\int_{\mathbb{T}} f(\zeta) d \sigma_{w_{n}}(\zeta)\right\}=\int_{\mathbb{T}} f(\zeta) d \sigma_{w}(\zeta)
$$


Proof. Assume first that $\varphi(0)=0$. Since Cauchy kernels and their conjugates form a complete system in the space of all continuous functions, it suffices to consider the case when $f$ is a Cauchy kernel. In this case we obtain

$$
\lim _{n \rightarrow \infty} \frac{1}{1-\bar{w}_{n} \varphi(z)}=\frac{1}{1-\bar{w} \varphi(z)}
$$

If $\varphi(0) \neq 0$, let

$$
\varphi_{1}(z)=\frac{\varphi(z)-\varphi(0)}{1-\overline{\varphi(0)} \varphi(z)} .
$$

Then $\varphi_{1}(0)=0$ and the result holds for $\varphi$ by Corollary 1 .

4. Outer functions in the $\varphi$ - $p$ factorization. For $0<p \leq \infty$ and $f \in H^{p}$, let $f$ have the $\varphi-p$ factorization $f=h_{p} F_{p} \circ \varphi$. From [10] we know that if $f \in H^{p}$, then for $0<r \leq p \leq \infty$, the outer part $F_{r}$ is in $H^{p}$ and $\left\|F_{r}\right\|_{p} \leq\|f\|_{p}$. This follows from Aleksandrov's theorem (Theorem B) and Theorem $\mathrm{G}$ above. To make this section self-contained we present below this simple argument.

Since, for $f=h_{r} F_{r} \circ \varphi$,

$$
F_{r}(z)=\exp \left\{\frac{1}{r} \int_{\mathbb{T}} \frac{\zeta+z}{\zeta-z} \log \left(\int_{\mathbb{T}}|f(\tau)|^{r} d \sigma_{\zeta}(\tau)\right) d m(\zeta)\right\}
$$

it follows from Riesz's formula that for almost every $\zeta \in \mathbb{T}$,

$$
\left|F_{r}(\zeta)\right|=\left\{\int_{\mathbb{T}}|f(\tau)|^{r} d \sigma_{\zeta}(\tau)\right\}^{1 / r} .
$$

Therefore,

$$
\int_{\mathbb{T}}\left|F_{r}(\zeta)\right|^{p} d m(\zeta)=\int_{\mathbb{T}}\left\{\int_{\mathbb{T}}|f(\tau)|^{r} d \sigma_{\zeta}(\tau)\right\}^{p / r} d m(\zeta) .
$$

Since $f^{r} \in L^{p / r}(\mathbb{T})$, applying Hölder's inequality to the inside integral we obtain

$$
\begin{aligned}
\int_{\mathbb{T}}\left|F_{r}(\zeta)\right|^{p} d m(\zeta) & \leq \int_{\mathbb{T}}\left\{\int_{\mathbb{T}}|f(\tau)|^{p} d \sigma_{\zeta}(\tau)\right\}\left\{\frac{1+|\varphi(0)|}{1-|\varphi(0)|}\right\}^{(p-r) / r} d m(\zeta) \\
& =\left\{\frac{1+|\varphi(0)|}{1-|\varphi(0)|}\right\}^{(p-r) / r}\|f\|_{p}^{p},
\end{aligned}
$$

where the last equality follows from Aleksandrov's Theorem (Theorem B).

The following theorem shows that the boundary value of the $\varphi-2$ outer part, $F_{2}$, of a function $f$ is smooth if $f$ is smooth. 
Theorem 5. Assume $\varphi$ is an inner function with $\varphi(0)=0$. Let $f$ be analytic in $\mathbb{D}$ and $f \in C^{N}(\overline{\mathbb{D}})$ on the boundary, $N>3$. Then the $\varphi-2$ outer part of $f$ is in $C^{N-4}(\overline{\mathbb{D}})$.

Proof. Assume $f(z)=\sum_{n=0}^{\infty} a_{n} z^{n}$ and $\varphi(0)=0$. Then

$$
|f(z)|^{2}=\sum_{n=0}^{\infty} a_{n} \bar{a}_{n}+\sum_{k=1}^{\infty} \sum_{n=k}^{\infty}\left(a_{n} \bar{a}_{n-k} z^{k}+\bar{a}_{n} a_{n-k} \bar{z}^{k}\right) .
$$

If we let

$$
\alpha_{k}=\sum_{n=k}^{\infty} a_{n} \bar{a}_{n-k}, \quad \bar{\alpha}_{k}=\sum_{n=k}^{\infty} \bar{a}_{n} a_{n-k}
$$

then by Aleksandrov's theorem

$$
\begin{aligned}
\left|F_{2}(w)\right|^{2} & =\int_{\mathbb{T}}|f(\zeta)|^{2} d \sigma_{w}(\zeta) \\
& =\alpha_{0}+\sum_{j=1}^{\infty} w^{j}\left\{\sum_{k=j}^{\infty} \alpha_{k} \beta_{j, k}\right\}+\bar{w}^{j}\left\{\sum_{k=j}^{\infty} \bar{\alpha}_{k} \bar{\beta}_{j, k}\right\} \\
& =\alpha_{0}+\sum_{j=1}^{\infty}\left(\gamma_{j} w^{j}+\bar{\gamma}_{j} \bar{w}^{j}\right),
\end{aligned}
$$

where $\beta_{j, k}=\int_{\mathbb{T}} \overline{\varphi(\zeta)^{j}} \zeta^{k} d m(\zeta)$ and $\gamma_{j}=\sum_{k=j}^{\infty} \alpha_{k} \beta_{j, k}$. Since $f \in C^{N}(\overline{\mathbb{D}})$, $\left|a_{k}\right|=o\left(1 / k^{N}\right)$ and therefore $\left|\alpha_{k}\right|=o\left(1 / k^{N}\right)$. Since $\left|\beta_{j, k}\right| \leq 1$ it follows that

$$
\gamma_{j}=o\left(1 / j^{N-1}\right)
$$

and hence $\left|F_{2}(w)\right|^{2} \in C^{N-3}(\mathbb{T})$.

Assume $\left|F_{2}\right|>0$ on $\mathbb{T}$. Then (8) implies

$$
\log \left(F_{2}(z)\right)=\int_{\mathbb{T}} \frac{\zeta+z}{\zeta-z} \log \left|F_{2}(\zeta)\right| d m(\zeta)
$$

is in $C^{N-4}(\overline{\mathbb{D}})$ (see [5, p. 81]). Thus

$$
F_{2}(\zeta)=\exp \left\{\frac{1}{2} \int_{\mathbb{T}} \frac{\zeta+z}{\zeta-z} \log \left|F_{2}(\zeta)\right|^{2} d m(\zeta)\right\}
$$

is in $C^{N-4}(\overline{\mathbb{D}})$.

Now let $F_{2}(\zeta) \in C^{N-4}(\mathbb{T})$ be arbitrary. Choose $\left\{\Psi_{n}\right\}, \Psi_{n}>0$, such that the $\Psi_{n}$ converge in the $C^{N-3}(\mathbb{T})$ topology to $\left|F_{2}\right|^{2}$. Then

$$
\exp \left\{\int_{\mathbb{T}} \frac{\zeta+z}{\zeta-z} \log \left(\Psi_{n}(\zeta)\right) d m(\zeta)\right\}=\Psi_{n}(z)
$$

converges in $C^{N-4}(\overline{\mathbb{D}})$ to $F_{2}(z)$.

The next corollary is straightforward in view of the proof of Theorem 5 . 
Corollary 2. If $f \in C^{\infty}(\overline{\mathbb{D}})$, then the $\varphi$-2 inner part of $f$ is in $C^{\infty}(\overline{\mathbb{D}})$.

If $f$ is analytic in the closed disk then the Taylor coefficients of $f$ decay exponentially. The argument of Theorem 5 shows that the Taylor coefficients of $\left|F_{2}\right|^{2}$ also decay exponentially. Thus we have the following corollary.

COROLlary 3. If $f$ is analytic in the closed unit disk then the $\varphi-2$ inner part of $f$ is analytic in the closed unit disk.

If $f$ is a polynomial and $\varphi(0)=0$ then we can say more.

Theorem 6. Assume $f$ is a polynomial and $\varphi(0)=0$. Then the $\varphi-2$ outer part of $f$ is a polynomial.

Proof. If $\varphi(0)=0$ one can deduce from (4) that $\left|F_{2}\right|^{2}$ is a symmetric trigonometric polynomial. Let $Q(\zeta)=\left|F_{2}(\zeta)\right|^{2}$. We show that there exists a polynomial $R(z)$ such that $|R(\zeta)|^{2}=Q(\zeta)$ on $\mathbb{T}$.

Since $Q$ is symmetric,

$$
Q(\zeta)=A_{-J} \bar{\zeta}^{J}+\ldots+A_{0}+\ldots+A_{J} \zeta^{J} \geq 0
$$

where $A_{J} \neq 0$ and $A_{-j}=\bar{A}_{j}$.

Now

$$
Q(\zeta)=\bar{\zeta}^{J}\left(A_{-J}+A_{-J+1} \zeta+\ldots+A_{j} \zeta^{2 J}\right) .
$$

Let

$$
q(\zeta)=A_{-J}+A_{-J+1} \zeta+\ldots+A_{j} \zeta^{2 J} .
$$

Assume $a$ satisfies $0<|a| \leq 1$ and $q(a)=0$. Then also $\overline{q(a)}=\bar{a}^{2 J} q(1 / \bar{a})$ $=0$. Hence, $1 / \bar{a}$ is also a root of $q$ outside the unit circle. Now let $a_{1}, \ldots, a_{J}$ be the roots of $q$ inside the unit circle and let $b_{j}=1 / \bar{a}_{j}$ be the corresponding roots outside the unit circle.

Write $r_{1}(z)=\prod_{j=1}^{J}\left(z-a_{j}\right)$ and $r_{2}(z)=\prod_{j=1}^{J}\left(z-b_{j}\right)$. Now $q(z)=$ $A_{J} r_{1}(z) r_{2}(z)$, and on the unit circle

$$
r_{2}(z)=\frac{(-1)^{J}}{\bar{z}^{J} \prod \bar{a}_{j}} \overline{r_{1}(z)}
$$

Since $Q(\zeta) \geq 0$ on $\mathbb{T}$, it follows that $A_{J}(-1)^{J} / \prod\left(\bar{a}_{j}\right)>0$. Define the polynomials

$$
R_{1}(z)=\sqrt{\frac{A_{J}(-1)^{J}}{\prod \bar{a}_{j}}} \prod_{j=1}^{J}\left(z-a_{J}\right), \quad R_{2}(z)=\sqrt{\frac{A_{J}(-1)^{J}}{\prod \bar{a}_{j}}} \prod_{j=1}^{J}\left(z-\frac{1}{\bar{a}_{J}}\right) .
$$

Then, on the unit circle,

$$
Q(z)=\bar{z}^{J} R_{1}(z) R_{2}(z)=R_{2}(z) \overline{R_{2}(z)}=\left|R_{2}(z)\right|^{2} .
$$


5. Bounded functions with unbounded $\varphi-p$ inner part. Here, we give a proof of Theorem 2. Our construction refines the one given in [10]. We start with the following lemma.

Lemma 3. Let $\varphi$ be an inner function having the property that $\sigma_{w}$ measures are continuous measures for all $w \in \mathbb{T}$. Fix $p_{0}>0$. There exists an $f$ in the unit ball of $H^{\infty}, f: \mathbb{D} \rightarrow \mathbb{D}$, such that for all $p \geq p_{0}$ the $\varphi$-p inner part of $f$ is not in $H^{q}$ for any $q>p$.

Proof. Assume first that $\varphi(0)=0$ so that the $\sigma_{w}$ are probability measures. Let $E_{w}=\varphi^{-1}(\{w\})$. Let $\left\{I_{n}\right\}$ be a set of arcs, $n=2,3, \ldots$, with the following properties:

(i) $I_{n} \cap I_{m}=\emptyset$ unless $n=m$.

(ii) $\left|I_{n}\right|=c /\left(n(\log n)^{3}\right)$ ( $c$ a normalizing constant independent of $n$ so that $\left.\sum\left|I_{n}\right| \leq 1\right)$.

Let $V_{n}=\varphi^{-1}\left(I_{n}\right)=\bigcup_{w \in I_{n}} E_{w}$. Then $V_{n} \cap V_{m}=\emptyset$ for $n \neq m$. Furthermore, the Lebesgue measure $\left|V_{n}\right|$ is positive and by (5),

$$
\left|V_{n}\right|=\left|I_{n}\right| \text {. }
$$

For each $w \in I_{n}$, define $J_{w}=\left(1, e^{i \theta(w)}\right)$ where $\sigma_{w}\left(J_{w}\right)=1 / n^{p_{0}}$. This can be done since the $\sigma_{w}$ are assumed to be continuous. Define

$$
U_{n}=\bigcup_{w \in I_{n}}\left(J_{w} \cap E_{w}\right) .
$$

Note that the terms $J_{w} \cap E_{w}$ are disjoint. Furthermore, $U_{n} \cap U_{m}=\emptyset$ for $n \neq m$ and $U_{n} \subset V_{n}$. Therefore,

$$
\sigma_{w}\left(U_{n}\right)= \begin{cases}1 / n^{p_{0}}, & w \in I_{n}, \\ 0, & w \in \mathbb{T} \backslash I_{n} .\end{cases}
$$

Thus, as in (5), $U_{n}$ has positive Lebesgue measure,

$$
\left|U_{n}\right|=\frac{1}{n^{p_{0}}}\left|I_{n}\right|=\frac{c}{n^{p_{0}} n(\log n)^{3}} .
$$

Define the following function on $\mathbb{T}$ :

$$
\varrho(w)= \begin{cases}1 & \text { if } w \in U_{n} \\ 1 / n & \text { if } w \in V_{n} \backslash U_{n}, \\ 1 & \text { if } w \in \mathbb{T} \backslash \bigcup_{n=2}^{\infty} V_{n} .\end{cases}
$$

Since $\varrho \in L^{\infty}(\mathbb{T})$ and

$$
\int_{\mathbb{T}}|\log (\varrho(w))| d m(w)=c \sum_{n=2}^{\infty} \frac{1-1 / n^{p_{0}}}{n(\log n)^{2}}<\infty,
$$

there exists an $f \in H^{\infty}$ such that $|f(\zeta)|=\varrho(\zeta)$ for almost every $\zeta \in \mathbb{T}$. 
Let $f=h_{p} F_{p} \circ \varphi$ be the $\varphi$-p factorization of $f$. For $w \in I_{n}$,

$$
\left|F_{p}(w)\right|=\left\{\int_{E_{w}}|f(\zeta)|^{p} d \sigma_{w}(\zeta)\right\}^{1 / p}=\left\{\frac{1}{n^{p}}\left(1-\frac{1}{n^{p_{0}}}\right)+\frac{1}{n^{p_{0}}}\right\}^{1 / p} .
$$

Since $p \geq p_{0}$, it follows immediately from (11) that

$$
\frac{1}{n^{p_{0} / p}} \leq\left|F_{p}(w)\right| \leq \frac{2}{n^{p_{0} / p}} .
$$

The functions $f, h_{p}$, and $F_{p} \circ \varphi$ have radial limits almost everywhere. For all $\zeta \in U_{n}$ for which these limits exist,

$$
\left|h_{p}(\zeta)\right|=\frac{\varrho(\zeta)}{\left|F_{p}(\varphi(\zeta))\right|} \approx n^{p_{0} / p} \quad \text { if } p \geq p_{0} .
$$

Now, if $q>p$,

$$
\int_{\mathbb{T}}\left|h_{p}(\zeta)\right|^{q} d m(\zeta) \geq \sum_{n=2}^{\infty} \int_{U_{n}}\left|h_{p}(\zeta)\right|^{q} d m(\zeta) \geq \sum_{n=2}^{\infty} \frac{n^{p_{0} q / p} c}{n^{p_{0}} n(\log n)^{3}}
$$

The series above diverges since $q>p$.

If the $\sigma_{w}$ are not probability measures, then by (6) the conclusions of (10), (12), and (13) hold.

Proof of Theorem 2. Without loss of generality assume that the $\sigma_{w}$ are probability measures. Let $\left\{I_{n k}\right\}$ be a set of arcs, $n=2,3, \ldots, k=$ $0,1, \ldots$, which have the following properties:

(i) $I_{n k} \cap I_{m l}=\emptyset$ unless $n=m$ and $k=l$,

(ii) $\left|I_{n k}\right|=c_{k} /\left(n(\log n)^{3}\right)$ where $\left\{c_{k}\right\}$ is a sequence of positive numbers such that

$$
\sum_{k=0}^{\infty} c_{k}=\left(\sum_{n=2}^{\infty} \frac{1}{n(\log n)^{3}}\right)^{-1} .
$$

Let $V_{n k}=\varphi^{-1}\left(I_{n k}\right)$. The $V_{n k}$ are disjoint subsets of $\mathbb{T}$ with positive Lebesgue measure $\left|I_{n k}\right|$.

Now we iterate the construction of Lemma 1. For each $w \in I_{n k}$ let $J_{w}=$ $\left(1, e^{i \theta(w)}\right)$ be the arc so that $\sigma_{w}\left(J_{w}\right)=1 / n^{p_{k}}$ where $\left\{p_{k}\right\}$ is a decreasing sequence of numbers such that $\lim _{k \rightarrow \infty} p_{k}=0$. Define

$$
U_{n k}=\bigcup_{w \in I_{n k}}\left(J_{w} \cap E_{w}\right) .
$$

A computation similar to (5) shows that $U_{n k}$ has positive Lebesgue measure and

$$
\int_{\mathbb{T}} \chi_{U_{n k}}(\zeta) d m(\zeta)=\frac{c_{k}}{n^{p_{k}} n(\log n)^{3}} .
$$


Define a function $\varrho$ similar to $(9)$ on $\mathbb{T}$ by

$$
\varrho(w)= \begin{cases}1, & w \in U_{n k}, \\ 1 / n, & w \in V_{n k} \backslash U_{n k}, \\ 1, & w \in \mathbb{T} \backslash \bigcup_{n, k} V_{n k} .\end{cases}
$$

The function $\varrho$ is bounded and positive on $\mathbb{T}$. Furthermore $|\log \varrho|$ is in $L^{1}(\mathbb{T})$ since

$$
\begin{aligned}
\int_{\mathbb{T}}|\log \varrho(w)| d m(w) & =\sum_{k=0}^{\infty} \sum_{n=2}^{\infty} \frac{c_{k}(\log n)\left(1-1 / n^{p_{k}}\right)}{n(\log n)^{3}} \\
& \leq \sum_{k=0}^{\infty} c_{k} \sum_{n=2}^{\infty} \frac{1}{n(\log n)^{2}} \\
& =\left(\sum_{k=0}^{\infty} c_{k}\right)\left(\sum_{n=2}^{\infty} \frac{1}{n(\log n)^{2}}\right)<\infty .
\end{aligned}
$$

Again, there exists a function $f$, bounded and analytic in $\mathbb{D}$, such that $|f|$ coincides with $\varrho$ almost everywhere on $\mathbb{T}$.

Let $f=h_{p} F_{p} \circ \varphi$ be the $\varphi$-p factorization of $f$. For almost every $w \in I_{n k}$,

$$
\left|F_{p}(w)\right|=\left\{\int_{E_{w}}|f(\zeta)|^{p} d \sigma_{w}(\zeta)\right\}^{1 / p}=\left\{\frac{1}{n^{p}}\left(1-\frac{1}{n^{p_{k}}}\right)+\frac{1}{n^{p_{k}}}\right\}^{1 / p} .
$$

Similar to (12), the following estimates hold:

$$
\begin{aligned}
\frac{1}{n}\left\{1-\frac{1}{n^{p_{k}}}\right\}^{1 / p} & \leq\left|F_{p}(w)\right| \leq \frac{2}{n} \quad & & \text { for } p \leq p_{k}, \\
\frac{1}{n^{p_{k} / p}} & \leq\left|F_{p}(w)\right| \leq \frac{2^{1 / p}}{n^{p_{k} / p}} & & \text { for } p>p_{k} .
\end{aligned}
$$

The set of points $\zeta_{n k} \in U_{n k}$ where $\varphi, f, F_{p} \circ \varphi$, and $h_{p}$ all have radial limits has measure $\left|U_{n k}\right|$. For all such $\zeta_{n k}$,

$$
\left|h_{p}\left(\zeta_{n k}\right)\right|=\frac{\varrho\left(\zeta_{n k}\right)}{\left|F_{p}\left(\varphi\left(\zeta_{n k}\right)\right)\right|} \approx n^{p_{k} / p}
$$

for all $p_{k}<p$. Given $p \in(0, \infty)$, for $p_{k}<p$ we have

$$
\int_{\mathbb{T}}\left|h_{p}(\zeta)\right|^{q} d m(\zeta) \geq \sum_{n=2}^{\infty} \frac{c_{k} n^{p_{k} q / p}}{n^{p_{k}} n(\log n)^{3}} .
$$

The series on the right hand side diverges if $q>p$.

The construction in Theorem 2 may not necessarily work for arbitrary $\varphi$. We need a polynomial rate of decrease of the $\sigma_{w}\left(J_{w}\right)$. The following example 
shows that the construction used in the proof of Theorem 2 works for the singular inner function determined by a point mass at 1 .

EXAMPLE. Let $\varphi(\zeta)=e^{(\zeta+1) /(\zeta-1)}$. If $w \in \mathbb{T}$, then $\varphi^{-1}(w)=\left\{\zeta_{k}\right\}$ where $\zeta_{k} \in \mathbb{T}$ and

$$
\log w+2 \pi i k=\frac{\zeta_{k}+1}{\zeta_{k}-1},
$$

and solving for $\zeta_{k}$ yields

$$
\zeta_{k}=1+\frac{2}{\log w+2 \pi i k-1} .
$$

If $\zeta \neq 1$ and $\zeta \in \mathbb{T}$, then $\varphi$ is analytically extendable through $\zeta$. Therefore,

$$
\sigma\left(\zeta_{k}\right)=\frac{1}{\left|\varphi^{\prime}\left(\zeta_{k}\right)\right|}=\frac{\left|\zeta_{k}-1\right|^{2}}{2}=\frac{2}{|\log w+2 \pi i k-1|^{2}} \sim O\left(\frac{1}{k^{2}}\right) .
$$

Therefore, on the arc $\left(1, \zeta_{N}\right)$,

$$
\sigma_{w}\left(\left(1, \zeta_{N}\right)\right)=\sum_{k=N}^{\infty} \frac{2}{|\log w+2 \pi i k-1|^{2}} \sim O\left(\frac{1}{N}\right) .
$$

The conclusion of Theorem 2 will now follow if the $J_{w}=\left(1, e^{i \theta(w)}\right)$ are chosen so that

$$
\sigma_{w}\left(J_{w}\right) \sim 1 / n^{p_{k}} .
$$

This can always be done since for each $n$ and $p_{k}$ there exists an integer $N$ so that $1 /(N+1) \leq 1 / n^{p_{k}} \leq 1 / N$.

\section{The $\varphi-p$ factorization of a function analytically extendable through the spectrum of $\varphi$}

Proof of Theorem 1. We know that if $\varphi$ is a finite Blaschke product, then $h_{p}$ is bounded ([6]). Hence, we assume $\varphi$ is not a finite Blaschke product. Suppose $f$ to be analytically extendable through the spectrum of $\varphi$. Write $f=h_{p} F_{p} \circ \varphi$ for the $\varphi$-p factorization of $f$ and assume that $h_{p}$ is unbounded. Define $E$ to be the set of all $\zeta$ on the unit circle where $\varphi$ has unimodular nontangential limits. Then there exists a sequence $\left\{w_{k}\right\} \subset E$ such that $\left|h_{p}\left(w_{k}\right)\right|>k$. Without loss of generality we may assume $\lim _{k \rightarrow \infty} w_{k}=w_{0}$. From Theorem $\mathrm{F}$ it follows that $w_{0} \in \Sigma_{\varphi} \cap \mathbb{T}$. Define

$$
O_{\delta}=\left\{\zeta \in \mathbb{T}: d\left(\zeta, \Sigma_{\varphi} \cap \mathbb{T}\right)<\delta\right\} \quad \text { where } \quad d\left(\zeta, \Sigma_{\varphi} \cap \mathbb{T}\right)=\inf _{w \in \Sigma_{\varphi} \cap \mathbb{T}}|\zeta-w| .
$$

Since $f$ is analytically extendable in a neighborhood of $\Sigma_{\varphi} \cap \mathbb{T}$, there exists an $\varepsilon>0$ such that $f$ is analytic in $\bar{O}_{2 \varepsilon}$. Without loss of generality, we can assume $w_{k} \in O_{\varepsilon}$. Now,

$$
\left|h_{p}\left(w_{k}\right)\right|=\frac{\left|f\left(w_{k}\right)\right|}{\left|F_{p}\left(\varphi\left(w_{k}\right)\right)\right|} .
$$


Since $f$ is analytic in $O_{2 \varepsilon}, f$ is bounded in $O_{\varepsilon}$. Without loss of generality, we can assume that $|f| \leq 1$ on $O_{\varepsilon}$. Therefore, by Theorem G,

$$
\left|F_{p}\left(\varphi\left(w_{k}\right)\right)\right|=\left\{\int_{\mathbb{T}}|f(\zeta)|^{p} d \sigma_{\varphi\left(w_{k}\right)}(\zeta)\right\}^{1 / p} \leq \frac{1}{k} .
$$

Let $\tau_{0}$ be a limit point of $\varphi\left(w_{k}\right)=\tau_{k}$. We claim that

$$
\left\{\int_{O_{\varepsilon}}|f(\zeta)|^{p} d \sigma_{\tau_{0}}(\zeta)\right\}^{1 / p}=0
$$

To prove this, let

$$
A=\bar{O}_{\varepsilon}, \quad B=\mathbb{T} \backslash O_{2 \varepsilon} .
$$

Since $f$ is analytically extendable through $O_{2 \varepsilon}$, by Urysohn's lemma there exists a continuous function $\widehat{f}$ such that $|\widehat{f}(\zeta)| \leq|f(\zeta)|$ and

$$
\widehat{f}(\zeta)= \begin{cases}f(\zeta), & \zeta \in A \\ 0, & \zeta \in B\end{cases}
$$

Therefore, by Lemma 2 we have

$$
\begin{aligned}
\int_{O_{\varepsilon}}|f|^{p} d \sigma_{\tau_{0}} & =\int_{O_{\varepsilon}}|\widehat{f}|^{p} d \sigma_{\tau_{0}} \\
& \leq \int_{O_{2 \varepsilon}}|\widehat{f}|^{p} d \sigma_{\tau_{0}}=\int_{\mathbb{T}}|\widehat{f}|^{p} d \sigma_{\tau_{0}}=\lim _{k \rightarrow \infty} \int_{\mathbb{T}}|\widehat{f}|^{p} d \sigma_{\tau_{k}} \\
& \leq \lim _{k \rightarrow \infty} \int_{\mathbb{T}}|f|^{p} d \sigma_{\tau_{k}}=0 .
\end{aligned}
$$

Thus $\left.f\right|_{O_{\varepsilon}}=0$ almost everywhere $\sigma_{\tau_{0}}$. Since $f$ is analytically extendable through $O_{\varepsilon}, f$ has only a finite number of zeros in $O_{\varepsilon}$, which implies $E_{\tau_{0}}$ is finite on $O_{\varepsilon}$.

Let $\zeta \in \mathbb{T} \backslash O_{\varepsilon}$. If $\varphi$ is analytically extendable through an $\operatorname{arc} I \subset \mathbb{T}$, then $\varphi$ has a nonvanishing derivative on $I$. Therefore, since $\varphi$ is analytically extendable through $\mathbb{T} \backslash \Sigma_{\varphi}$, there exists a neighborhood $U_{\zeta}$ of $\zeta$ such that $\operatorname{card}\left(E_{\tau_{0}} \cap U_{\zeta}\right) \leq 1$. The open sets $\left\{U_{\zeta}\right\}$ cover $\mathbb{T} \backslash O_{\varepsilon}$. There exists a finite subcover $\left\{U_{\zeta_{i}}\right\}_{i=1}^{n}$. Thus $E_{\tau_{0}}$ is finite in $\mathbb{T} \backslash O_{\varepsilon}$. Now, since $E_{\tau_{0}}$ is finite in $\left\{\mathbb{T} \backslash O_{\varepsilon}\right\} \cup O_{\varepsilon}=\mathbb{T}, \varphi$ is a finite Blaschke product, a contradiction.

REMARK. If there does not exist an $\varepsilon$ so that the set $B$ is empty, then the function $f$ is analytic in the closed unit disk and the integral in (18) can be taken over the entire unit circle (see the argument immediately preceding the statement of Theorem 2).

7. $\varphi$ - $p$ factorization for the disk algebra. The following lemma is needed for the proof of Theorem 3 . 
Lemma 4. Assume there exists a point $w \in \mathbb{T}$ and an arc $I \subset \mathbb{T}$ such that $E_{w}$ is dense in $I$. Then the support of $\sigma_{w}$ is dense in $I$ for all $w \in \mathbb{T}$.

Proof. Assume that $E_{w_{1}}$ is not dense in I. That is, $I \nsubseteq \operatorname{supp}\left(\sigma_{w_{1}}\right)$. Then $\varphi$ has an analytic continuation through an arc $I_{1} \subset I$, which implies $\left\{\varphi^{-1}(w)\right\}$ is nowhere dense in $I_{1}$.

Proof of Theorem 3. Without loss of generality assume $|f(\zeta)| \leq 1$. Assume there exists a sequence $\left\{w_{n}\right\}$ so that $\left|h_{p}\left(w_{n}\right)\right|>2^{n}$. If $f=h_{p} F_{p} \circ \varphi$ then

$$
\left|h_{p}\left(w_{n}\right)\right|=\frac{\left|f\left(w_{n}\right)\right|}{\left|F_{p}\left(\varphi\left(w_{n}\right)\right)\right|}=\frac{\left|f\left(w_{n}\right)\right|}{\left\{\int_{\mathbb{T}}|f(\zeta)|^{p} d \sigma_{\varphi\left(w_{n}\right)}(\zeta)\right\}^{1 / p}} .
$$

Let

$$
A_{n}=\{w \in \mathbb{T}:|f(w)|>1 / n\} .
$$

Then

$$
\sigma_{\varphi\left(w_{n}\right)}\left(A_{m}\right)<m^{p} / 2^{n p} .
$$

There exists a subsequence $\left\{w_{n_{k}}\right\}$ so that $\lim _{n_{k} \rightarrow \infty} \varphi\left(w_{n_{k}}\right)=w_{0}$. By Lemma $2, \sigma_{w_{0}}\left(A_{m}\right)=0$. Furthermore,

$$
\sigma_{w_{0}}\left(\bigcup_{n} A_{n}\right) \leq \sum_{m} \sigma_{w_{0}}\left(A_{m}\right)=0
$$

Now,

$$
\sigma_{w_{0}}\left(I \backslash\left\{\bigcup_{n=1}^{\infty} A_{n}\right\}\right)=\sigma_{w_{0}}(I \cap\{\zeta \in \mathbb{T}: f(\zeta)=0\})=\sigma_{w_{0}}(I) .
$$

The set $\{\zeta \in \mathbb{T}: f(\zeta)=0\}$ is dense in $I$. Otherwise, there exists some arc $I_{0}$ which is in the complement of $\{\zeta \in \mathbb{T}: f(\zeta)=0\}$ in $I$. The support of the measure $\sigma_{w_{0}}$ is dense in $I$, hence it follows that $\sigma_{w_{0}}\left(I_{0}\right)>0$. But this contradicts the fact that $\sigma_{w}\left(\bigcup_{n=1}^{\infty} A_{n}\right)=0$.

Since $f$ is continuous on $\mathbb{T},\{w \in \mathbb{T}: f(\zeta)=0\}$ dense in $I$ implies that $f \equiv 0$, a contradiction. We can now conclude that $h_{p}$ is bounded.

Proof of Theorem 4. Since

$$
\bar{E}_{w_{0}}=E_{w_{0}} \cup\left\{\Sigma_{\varphi} \cap \mathbb{T}\right\},
$$

$\left|\bar{E}_{w_{0}}\right|=0$. It follows from Aleksandrov's theorem that

$$
0=\int_{\mathbb{T}} \chi_{\bar{E}_{w_{0}}}(\zeta) d m(\zeta)=\iint_{\mathbb{T}} \chi_{\bar{E}_{w_{0}}}(\zeta) d \sigma_{w}(\zeta) d m(w) .
$$

This implies that for almost all $w \in \mathbb{T}$,

$$
\sigma_{w}\left(\bar{E}_{w_{0}}\right)=\int_{\mathbb{T}} \chi_{\bar{E}_{w_{0}}}(\zeta) d \sigma_{w}(\zeta)=0 .
$$


Let $\left\{w_{k}\right\}$ be a sequence in $\mathbb{T}$ such that

$$
\lim _{k \rightarrow \infty} w_{k}=w_{0} \quad \text { and } \quad \sigma_{w_{k}}\left(\bar{E}_{w_{0}}\right)=0 .
$$

Let

$$
C=\left(\bigcup_{k} E_{w_{k}}\right) \cup \bar{E}_{w_{0}} .
$$

Claim. The set $C$ is a closed subset of $\mathbb{T}$.

Let $\zeta$ be a limit point of $C$. Then there exists a sequence $\left\{\zeta_{n}\right\}$ in $C$ converging to $\zeta$. We have either (i) or (ii) below:

(i) The points $\zeta_{n}$ lie in some $E_{w_{k}}$ for an infinite number of $n$. In this case,

$$
\zeta \in \Sigma_{\varphi} \cap \mathbb{T} \subset \bar{E}_{w_{0}} .
$$

(ii) Each $E_{w_{k}}$ contains at most a finite number of the $\zeta_{n}$. If $\varphi$ is continuous at $\zeta$ then

$$
\lim _{n \rightarrow \infty} \varphi\left(\zeta_{n}\right)=\lim _{k \rightarrow \infty} w_{k_{n}}=w_{0}=\varphi(\zeta) .
$$

In this case $\zeta \in E_{w_{0}} \subset \bar{E}_{w_{0}}$. If $\varphi$ is not continuous at $\zeta$ then $\zeta \in \Sigma_{\varphi} \cap \mathbb{T} \subset$ $\bar{E}_{w_{0}}$. Thus $C$ is closed in $\mathbb{T}$. Furthermore,

$$
m(C)=\sum_{k=1}^{\infty} m\left(E_{w_{k}}\right)+m\left(\bar{E}_{w_{0}}\right)=0 .
$$

Since $E_{w_{k}}$ is not finite, and $E_{w}$ consists of the point masses of $\sigma_{w}$, there exists a $\zeta_{k} \in E_{w_{k}}$ so that $\sigma_{w_{k}}\left(\zeta_{k}\right) \leq 1 / k$. Define the following function $\varrho$ on $C$ :

$$
\varrho(\zeta)= \begin{cases}1 / k & \text { if } \zeta=\zeta_{k}, \\ 0 & \text { if } \zeta \in C \backslash\left\{\zeta_{k}\right\}_{k=0}^{\infty} .\end{cases}
$$

It is easily seen that the function $\varrho$ is continuous on $C$. Since $\varrho$ is defined on a set of measure zero, by the Rudin-Carleson theorem there exists a function $f \in \mathbf{A}$ such that $f(\zeta)=\varrho(\zeta)$ for $\zeta \in C$. If $f=h_{p} F_{p} \circ \varphi$, then

$$
\begin{aligned}
\left|F_{p}\left(w_{k}\right)\right| & =\left\{\int_{\mathbb{T}}|f(\zeta)|^{p} d \sigma_{w_{k}}(\zeta)\right\}^{1 / p}=\left\{\frac{1}{k^{p}} \sigma_{w_{k}}\left(\zeta_{k}\right)\right\}^{1 / p} \\
& \leq \frac{1}{k^{1+1 / p}}
\end{aligned}
$$

It follows that

$$
\left|h_{p}\left(\zeta_{k}\right)\right|=\frac{\left|f\left(\zeta_{k}\right)\right|}{\left|F_{p}\left(w_{k}\right)\right|} \geq k^{1 / p},
$$

from which we conclude that $\lim _{k \rightarrow \infty} h_{p}\left(\zeta_{k}\right)=\infty$. 
8. Concluding remarks. It is possible to extend the result of Theorem 5 to any $p>0$. The conclusion for given $f \in C^{N}(\overline{\mathbb{D}}) \cap H^{p}(\mathbb{D})$ follows by considering the canonical factorization of $f$ into its inner and outer part, $f(z)=g(z) F(z)$. If $F=h_{p} \widetilde{F}_{p} \circ \varphi$ is the $\varphi-p$ factorization of $F$, then

$$
F_{p}^{p / 2}=h_{p}^{p / 2} \widetilde{F}_{p}^{p / 2} \circ \varphi
$$

is the $\varphi-2$ factorization of $F_{p}^{p / 2}$. Suppose $F \neq 0$ on the boundary. Since $F \in C^{N}(\overline{\mathbb{D}})$, it follows from Theorem 5 that $\widetilde{F}_{p}^{p / 2} \in C^{N-4}(\overline{\mathbb{D}})$. Since $\widetilde{F}_{p} \neq 0$ on $\mathbb{T}$, it follows that $\widetilde{F}_{p} \in C^{N-4}(\overline{\mathbb{D}})$. The conclusion of Theorem 5 now follows since $f=\left\{g h_{p}\right\} \widetilde{F}_{p} \circ \varphi$ is the $\varphi$-p factorization of $f$. If $F$ has zeros on $\mathbb{T}$ we may approximate $\widetilde{F}_{p}$ as before.

Given an inner function $\varphi$ and a function $f \in H^{p}$, we want to continue to investigate the $\varphi-p$ inner part. For instance, if we pose certain restrictions on $\varphi$, are there any other classes of functions that have bounded $\varphi-p$ inner part? On other hand, can we find conditions for $\varphi$-other than continuity of the measures - which will ensure the existence of an $f$ which has an unbounded $\varphi-p$ inner part? From the Example in Section 5, we see that the measures $\sigma_{w}$ need not be continuous for the construction in Theorem 1 to work. We pose the following conjecture.

CONJECTURE 1. The conclusion of Theorem 2 holds for an arbitrary inner function which is not a finite Blaschke product. That is, for every $\varphi$ inner, there exists a function $f$ whose $\varphi$-p outer part is not in $L^{q}$ for any $q>p$.

\section{References}

[1] A. B. Aleksandrov, Multiplicity of boundary values of inner functions, Izv. Akad. Nauk. Armyan. SSR Ser. Mat. 22 (1987), 490-503 (in Russian).

[2] C. Carathéodory, Theory of Functions, Chelsea, New York, 1958.

[3] D. N. Clark, One dimensional perturbations of restricted shifts, J. Anal. Math. 25 (1972), 169-191.

[4] K. Hoffman, Banach Spaces of Analytic Functions, Dover, New York, 1988.

[5] Y. Katznelson, An Introduction to Harmonic Analysis, Dover, New York, 1968.

[6] T. Lance and M. Stessin, Multiplication invariant subspaces of Hardy spaces, Canad. J. Math. 49 (1997), 100-118.

[7] N. K. Nikol'skiu, Treatise on the Shift Operator: Spectral Function Theory, Springer, New York, 1986.

[8] A. G. Poltoratskiı̌, On the distributions of boundary values of Cauchy integrals, Proc. Amer. Math. Soc. 124 (1996), 2455-2463.

[9] J. H. Shapiro, Composition Operators and Classical Function Theory, Springer, New York, 1993. 
[10] M. Stessin and K. Zhu, Generalized factorization in Hardy spaces and the commutant of Toeplitz operators, Canad. J. Math., to appear.

Department of Mathematics

University of North Carolina

346 Phillips Hall 3250

Chapel Hill, NC 27599-3250, U.S.A.

E-mail: cima@email.unc.edu

Department of Mathematics and Statistics

SUNY at Albany

Albany, NY 12222, U.S.A.

E-mail: stessin@albany.edu
Department of Mathematics, Computer Science, and Statistics SUNY at Oneonta Oneonta, NY 13820, U.S.A. E-mail: kazasa@oneonta.edu

Received May 3, 2002

Revised version March 10, 2003 